\title{
PERSPECTIVES
}

\section{Disruptive Influences on Research in Academic Pathology Departments}

\section{Proposed Changes to the Common Rule Governing Informed Consent for Research Use of Biospecimens and to Rules Governing Return of Research Results}

\author{
Mark E. Sobel* and Jennifer C. Dreyfus
}

From the American Society for Investigative Pathology, ${ }^{*}$ Bethesda; and the Dreyfus Consulting, $L L C,^{\dagger}$ Takoma Park, Maryland

Accepted for publication

November 3, 2016

Address correspondence to Mark E. Sobel, M.D., Ph.D., American Society for Investigative Pathology, 9650 Rockville Pike, Ste E130, Bethesda, MD 20814. E-mail: mesobel@ asip.org.

\begin{abstract}
Academic pathology departments will be dramatically affected by proposed United States federal government regulatory initiatives. Pathology research will be substantially altered if proposed changes to the Common Rule (Code of Federal Regulations: Protection of Human Subjects title 45 CFR 46) and regulations governing the return of individual research results are approved and finalized, even more so now that the Precision Medicine initiative has been launched. Together, these changes are disruptive influences on academic pathology research as we know it, straining limited resources and compromising advances in diagnostic and academic pathology. Academic research pathologists will be challenged over the coming years and must demonstrate leadership to ensure the continued availability of and the ethical use of research pathology specimens. (Am J Pathol 2017, 187: 4-8; http://dx.doi.org/10.1016/ j.ajpath.2016.11.001)
\end{abstract}

\section{History}

The conduct of pathology research has been governed by a variety of ethical considerations. The 1974 National Research Act generated the first national bioethics committee in the United States-the National Commission for the Protection of Human Subjects of Biomedical and Behavioral Research. The Commission is best known for publishing The Belmont Report (1979), ${ }^{1}$ which established the key principles of respect for persons (autonomy), beneficence, and justice. These principles have their roots in the 1964 Declaration of Helsinki, ${ }^{2}$ which established the basis for research ethics.

In 1981, the Common Rule ${ }^{3}$ generated a baseline ethical standard for government-funded research in the United States, with nearly all academic institutions holding their researchers to these statements of rights regardless of funding source. The Common Rule applied to government-funded research from 15 federal agencies (later expanded to 17 and finally 19 agencies in 2015), and led to the requirement for informed consent for human subjects research and the generation of institutional review boards (IRBs) for the oversight of that research in the United States. The Common Rule was subsequently revised in 1991.

Beginning in the mid-1990s, the issue of biospecimen use in research and the Common Rule regulations were moved to the fore. When the National Center for Human Genome Research was launched, its Ethical, Legal, and Social Issues Working Group was tasked with proactively considering the

Supported by the American Society for Investigative Pathology.

Disclosures: M.E.S. is employed as the Executive Officer of the American Society for Investigative Pathology. J.C.D. is a Science Policy Consultant for the American Society for Investigative Pathology.

This article is partly based on a presentation given by M.E.S. at the Association of Pathology Chairs Annual Meeting in San Bernardo, California, on July 12, 2016. 
rights of subjects participating in research studies, including a recognition that an individual's biospecimens, whether obtained through clinical intervention (biopsy, surgical resection of diseased tissue, or body fluids for laboratory testing) or through a research study, should be included under the umbrella of human research subjects protections. A seminal Journal of the American Medical Association article in $1995^{4}$ raised concerns about lack of informed consent for research use of human biospecimens. A consortium of 17 national organizations responded in 1999 with recommendations for responsible conduct of research on human biospecimens. ${ }^{5}$

A central concept of the Common Rule is exemption from the regulations based on the definition of human subject: "A living individual about whom an investigator (whether professional or student) conducting research obtains data through intervention or interaction with the individual, or identifiable private information." ${ }^{33}$ As a result, several types of research fall outside the definition of human subjects research, including research conducted on deceased persons (ie, autopsy specimens), studies using publicly available information, and research using nonidentified biospecimens. Such research has been exempt from Common Rule regulatory oversight. Nonidentified biospecimens include socalled anonymous samples that were originally collected without any data that could reveal the identity of the donor (although they can be associated with some demographic data), as well as anonymized samples that were originally collected with an identifier but the identifier has since been permanently stripped from the sample, rendering it anonymous. Examples of identifiers include name, medical record chart number, and social security number. In contrast, the definition of an identified sample refers to a biospecimen that can be identified by any one person at any time or location. Coded or linked biospecimens are used in research with a random identifier developed to disconnect the specimen from identifiable information. However, if there is a method for linking the random identifier to the specimen that can be accessed by the researcher, even if under lock and key or encryption, the biospecimen is considered identifiable.

A key component of the Common Rule is the ability of an IRB to grant a waiver of informed consent if the proposed research meets four criteria: i) minimal risk, ii) respect for autonomy and the rights of the individual, iii) impracticability of obtaining consent, and iv) notification. Using an algorithm developed by the Office of Human Research Protections, IRBs generally follow a rule that informed consent is not waived for the use of identified samples, and is rarely, if ever, waived for coded (linked) samples.

A 2008 Guidance $^{6}$ from the Office of Human Research Protections, US Department of Health and Human Services, clarified that research on biological specimens is not considered human research if the following two criteria are met: the specimen was not collected specifically for a current proposed research project through an interaction or intervention with a living individual; and the investigator is not able to readily ascertain the identity of the individual(s). In essence, by signing an agreement that the researcher has no intention to break the code of a linked biospecimen or to discern the identity of the donor, a pathologist or other biomedical researcher may more readily obtain a waiver of informed consent from the IRB to conduct research using nonidentified biological specimens.

In 2011, two components of the US Department of Health and Human Services (Office of the Secretary and the Food and Drug Administration) issued an Advanced Notice of Proposed Rulemaking, ${ }^{7}$ proposing a variety of changes to update the Common Rule. A central premise of the Advanced Notice of Proposed Rulemaking was that the application of new technologies, such as next-generation sequencing, makes it possible for any sample to be identified and that there is, in fact, no such thing as an anonymous or nonidentified sample. Indeed, a research report by Gymrek et al $^{8}$ in 2013 demonstrated that sequencing a significant portion of the genome may result in the specimen being considered identifiable and therefore worthy of appropriate protections under human subjects research regulations. This research study further heightened concern as to whether the ability to inexpensively and rapidly sequence the genome of an individual from a single cell of a biospecimen nullifies the concept of an anonymous or anonymized sample.

No further regulatory action was taken for 4 years. In September 2015, the US federal agencies covered under the Common Rule, now 19 in number, jointly issued a Notice of Proposed Rulemaking (NPRM).9 Notably, the NPRM broadened the definition of a human subject to include nonidentified biospecimens. As a result, research use of nonidentified biospecimens would no longer be exempt from human subject research protections, as they are currently under the Common Rule. The NPRM also set such strict criteria for IRB waivers of informed consent that they would be granted only under rare circumstances.

\section{Response to the Common Rule NPRM}

By the end of the most recent comment period on the proposed regulatory changes, the US federal government had received well over 2100 comments from researchers, research institutions, research participants, the general public, and others. Currently, federal regulators are reviewing these comments and determining which, if any, will be incorporated into a final rule. As of the last update in May 2016, finalizing the proposed changes to the Common Rule remains on the Unified Agenda, ${ }^{10}$ and thus a topic of ongoing regulatory activity.

The 2015 NPRM $^{9}$ emphasized the concept of autonomy, recommending that research using nonidentified biospecimens would now be considered human subjects research requiring informed consent. The NPRM proposed 
extending the regulations to cover all clinical trials conducted in the United States, regardless of funding source. The proposed regulations outlined the use of broad consents for the storage and maintenance of biospecimens for future, unspecified secondary research studies. Once signed, such broad consents would allow biospecimens to be collected from an individual for 10 years and allow the use of those collected specimens indefinitely. Ten years after signing a broad consent, collection of biospecimens from a given individual would cease until a new consent was signed. Although there were many additional changes proposed in the NPRM, the above changes would have the most profound effect on research pathology, applying to both identified and nonidentified biospecimens.

The US federal government estimates that the cost burden for just the new broad consents for secondary use requirement would exceed $\$ 12$ billion over 10 years. ${ }^{9}$ In its November 23, 2015, comment letter on the NPRM (American Society for Investigative Pathology, http://www. asip.org/SciencePolicy/documents/ASIPCommentsNPRM CommonRule.pdf, last accessed November 2016), the American Society for Investigative Pathology reported that by its own analysis the federal calculations grossly underestimate the cost of the NPRM. According to the American Society for Investigative Pathology, the cost burden calculated by the US federal government included several faulty assumptions, including the following: i) assuming that only federal-wide assurance institutions will store and maintain biospecimens; ii) assuming that it takes only 5 minutes to obtain consent in a research setting and 10 minutes in a clinical setting; iii) assuming that research institutions would only need one full-time equivalent database administrator per institution to comply with the proposed regulation; and iv) assuming that there was no consideration of an allowance for development of robust databases that would track individual biospecimens, allowing individuals to consent for one type of biospecimen research but not another (eg, research may be conducted on blood but not on urine).

Speaking to the many issues in the NPRM, the National Academies of Sciences, Engineering, and Medicine released a report in $2015^{11}$ entitled Optimizing the Nation's Investment in Academic Research: A New Regulatory Framework for the $21^{\text {st }}$ Century: Part 1, which raised significant questions regarding how additional regulations affect the ability to conduct research, education, and scholarship where "the unintended cumulative effect of federal regulations undercuts the productivity of the research enterprise and diminishes the return of the federal investment in research."11 The initial National Academies of Sciences, Engineering, and Medicine report was expanded in 2016, ${ }^{12}$ recommending that the proposed changes to the Common Rule be withdrawn and that a new independent commission be appointed to review the framework for human subjects research.

The Council on Government Relations, along with the Association of Public and Land-Grant Universities, publicly released its analysis of the $>2100$ comments submitted in response to the NPRM in May, 2016 (Council on Government Relations, http://www.cogr.edu/COGR/files/ ccLibraryFiles/Filename/000000000346/Analysis\%20of\% 20Common\%20Rule\%20Comments.pdf, last accessed November 2016). Approximately $70 \%$ of the comments addressed the proposed changes to nonidentified biospecimen regulations. More than half of the general public and $80 \%$ of the top 30-ranked universities opposed the proposed changes.

It remains a possibility that the US federal government will release the final Common Rule with many of the problematic provisions outlined in the NPRM ${ }^{9}$ and highlighted by the National Academies of Sciences, Engineering, and Medicine. ${ }^{11,12}$ Alternatively, it is possible that the final recommendations will fall more along the lines of those proposed by the Secretary's Advisory Committee on Human Research Protections (SACHRP) in its posting of December 28, 2015. ${ }^{13}$ The SACHRP recommendations have struck a different balance between autonomy and the ethical conduct of research in support of scientific progress. More important, they do not support the broadened definition of a human subject to include nonidentified biospecimens and therefore would allow for the continued use of nonidentified biospecimens as exempt from human subjects research regulations. The SACHRP recommendations do not support the use of broad consents; however, they include other recommendations that minimize the burden placed on researchers.

\section{Return of Research Results}

A variety of factors have led to increasing support for sharing final study data and results with research participants and the general public. One useful way of reviewing return of results considerations is through the following general, yet somewhat overlapping, categories ${ }^{14,15}$ : i) return of incidental findings to subjects, ii) return of individual study results to subjects, iii) return of general study results to subjects, and iv) public release of study data. Recent opinions of SACHRP ${ }^{15}$ indicate its general support for returning study results in a wide variety of situations. They have posited a rebuttable presumption for return of results along with several reasons that are listed as potential justifications for not returning results, such as the following: i) potential for study bias; ii) significant administrative and/ or financial burden to the investigator, institution, or other involved parties; iii) unknown meaning and significance of the research results; iv) low importance or insignificance of the results; or v) results lacking validity or actionability. Under the rebuttable presumption framework, the burden is shifted to the researcher and research institution to indicate why results will not be returned. The recommendations of SACHRP $^{14}$ were issued in the form of a letter to the US Secretary of Health and Human Services and lack the strength of a guidance or regulation. Potentially, SACHRP recommendations could become the foundation for a future 
guidance and thus not be open for public comment, as required for proposed US federal regulations.

There continues to be disharmony between the Health Insurance Portability and Accountability Act and the Clinical Laboratory Improvement Act (CLIA). The US Center for Medicare and Medicaid Services interprets CLIA such that research findings returned to patients must be generated by a CLIA-certified laboratory. The US Center for Medicare and Medicaid Services has stated that returning research results from a non-CLIA laboratory violates the research exemption under CLIA. ${ }^{15}$ This in and of itself may be problematic because some cutting-edge technologies, particularly in the area of precision medicine, may be generated by a non-CLIA-certified laboratory. The US Center for Medicare and Medicaid Services interpretation of CLIA conflicts directly with the requirements of the Health Insurance Portability and Accountability Act, mandating the disclosure of individual results on request where the results are generated by a covered entity and fit within the definition of designated record set (typically broader than the general term of medical record). Most results generated by an academic pathology department would meet both of these requirements. The standards for return of research results are undergoing significant shifts, and academic departments of pathology should pay close attention to the shifting landscape.

\section{Safeguarding Pathology Resources}

Academic research pathologists are well positioned to assume leadership regarding the ethical use of biospecimens for research, providing stewardship and ensuring that these specimens remain a vital resource. Especially given the increasing focus on precision medicine and the sensitive nature of genetic information, donors will be more likely to provide tissue samples to researchers who engender trust. ${ }^{16}$ With appropriate measures of security and confidentiality, donor trust may be encouraged and research enhanced. A consortium of 17 national organizations has proposed ${ }^{5}$ that researchers should foster and support efforts to develop institutional policies that define how data confidentiality, integrity, security, and controlled access will be ensured.

Pathology researchers already work with IRBs, ensuring appropriate oversight of the donor consent process and maintenance of, access to, and use of pathology research resources, such as human biospecimens. Pathology departments also play a critical role in ensuring data confidentiality, integrity, security, and controlled access to information. The level of security should coincide with the sensitivity of the information, recognizing recent concerns about the technological ease of extracting genetic material from biospecimens. ${ }^{8}$ Such activities are vital to ensure the public's trust in the confidentiality and security of data generated from biospecimens.

Broad consents have been recognized as suitable to address rapid and/or unexpected developments in scientific research. ${ }^{17}$ If broad consent is sought, it should provide the donor with a general sense of possible research uses, including any risks that might be associated with current and future research. Where the possibility exists that future research may look at a significant portion of the genome, this action should be clearly specified within the donor consent. The consent should also address, as needed, the following: i) whether the donor may withdraw from future research (and how already obtained data will be handled); ii) risks, including unintended revelation of the donor's identity and the subsequent possible impact on the individual and associated family; iii) institutional efforts to protect the individual's privacy; iv) whether there will be financial benefit to the donor should a commercial product be developed; v) the level of personal information that will be coupled with the specimen; and vi) the potential for the specimen, as well as some or all of the associated personal information, to be shared with other researchers either within the institution or to an outside entity.

Technology has advanced, opening the potential for the reidentification of previously anonymous or anonymized tissue samples. ${ }^{9}$ In its November 23, 2015, comment letter on the NPRM (American Society for Investigative Pathology, http://www.asip.org/SciencePolicy/documents/ ASIPCommentsNPRMCommonRule.pdf, last accessed November 2016), the American Society for Investigative Pathology has proposed that unless specific donor consent is granted, all individuals working with research specimens should not attempt to identify previously unidentified samples. This prohibition includes using advanced technologies to identify a donor or accessing a research database and using it for purposes of identification.

Keeping the above considerations in mind, we recommend that holders of archived pathology specimens develop and institute policies regarding the use of specimens or digitized data that include data use agreements. A data use agreement could, for example, expressly prohibit researchers from seeking to reidentify a donor, which would foster security and confidentiality and engender public trust. Nonidentified human tissue samples can continue to be used provided that the current Office of Human Research Protections requirements ${ }^{6}$ are met and a data use agreement is in place. A data access committee may prove valuable in complying with this recommendation. In our opinion, data use agreements should, at a minimum, included the following components: i) a description of the intended research project; ii) limits on research use to the research project described in the agreement; iii) a description of the responsibilities of the researcher; iv) agreement of the researcher to not use the samples in an attempt to identify a donor unless expressly consented to; v) what happens to the data already obtained if the research subject wants to withdraw from the study; vi) non-transferability of samples to others not designated in the agreement; vii) delineation of requirements for security and encryption; viii) limits, if any, on the ability to publish any deidentified or identified donor 
genetic information; ix) obligation to report any inadvertent data release, breach of data security, or other incident; and $\mathrm{x})$ consequences if the terms of the agreement are violated.

We further recommend that all individuals working with human research biospecimens receive training in the applicable regulations. Relevant training may include information on the Health Information Technology for Economic and Clinical Health Act and the Health Insurance Portability and Accountability Act, as well as the Common Rule.

Funders of research, whether public or private, should recognize the researcher's obligation to respect individual privacy and the need for compliance with data access agreements. Ideally, policies on public access of data should balance the shared goal of advancing research with the researcher's obligation to ensure donor trust through ethical behavior. Publishers, genetic information databases, and other entities should develop guidelines that balance the need to respect individual privacy with the need to broadly share research data to allow for collaboration and replication. The guidelines should ensure consistency between the donor consent, data access agreements, and the potential uses of the data.

Pathology research will be dramatically affected on the basis of the proposed changes ${ }^{9}$ to the Common Rule, evolving thoughts on the return of research results, and the Precision Medicine Initiative. Together, these changes can be seen as potentially disruptive influences on academic pathology research as we know it, straining limited resources, compromising advances in diagnostic and academic pathology, and possibly transforming our discipline. Academic research pathologists will be challenged over the coming years and must demonstrate leadership, ensuring the ongoing availability of and the ethical use of research pathology specimens. Only through our ongoing commitment to stewardship will these biospecimens remain a vital resource for the betterment of humankind.

\section{Acknowledgment}

We thank the American Society for Investigative Pathology Research and Science Policy Committee (Chair, Daniel Remick, M.D., Boston University School of Medicine, and Chair-Elect, William Muller, M.D., Ph.D., Northwestern University School of Medicine) for formative discussions.

\section{References}

1. National Commission for the Protection of Human Subjects of Biomedical and Behavioral Research. The Belmont Report: Office of the Secretary Ethical Principles and Guidelines for the Protection of Human Subjects of Research. April 18, 1979. Available at http://www.hhs.gov/ohrp/ regulations-and-policy/belmont-report/index.html (accessed November 2016)

2. World Medical Association Declaration of Helsinki: Ethical Principles for Medical Research Involving Human Subjects, adopted by the 18th WMA General Assembly, Helsinki, Finland, June 1964. Available at http://www.wma.net/en/30publications/10policies/b3/ index.html (accessed November 2016)

3. Code of Federal Regulations: Protection of Human Subjects, title 45 CFR 46 (2009). Available at http://www.hhs.gov/ohrp/regulations-andpolicy/regulations/45-cfr-46/index.html (accessed November 2016)

4. Clayton EW, Steinberg KK, Khoury MJ, Thomson E, Andres L, Kahn MJ, Kopelman LM, Weiss JO: Informed consent for genetic research on stored tissue samples. JAMA 1995, 274:1786-1792

5. Grizzle W, Grody WW, Noll WW, Sobel ME, Stass SA, Trainer T, Travers H, Weedn V, Woodruff K: Recommended policies for uses of human tissue in research, education, and quality control. Arch Pathol Lab Med 1999, 123:296-300

6. Office of Human Research Protections. Guidance on Research Using Coded Private Information or Specimens, October 16, 2008. Available at http://www.hhs.gov/ohrp/regulations-and-policy/guidance/researchinvolving-coded-private-information/index.html (accessed November 2016)

7. Advanced Notice of Proposed Rulemaking. Federal Register Volume 76, Number 143, p, 44512-44531. (July 26, 2011). Available at https://www.gpo.gov/fdsys/pkg/FR-2011-07-26/html/2011-18792.htm (accessed November 2016)

8. Gymrek M, McGuire AL, Golan D, Halperin E, Erlich Y: Identifying personal genomes by surname inference. Science 2013, 339:321-324

9. Notice of Proposed Rulemaking. Federal Register Volume 80, Number 173, p. 53931-54061. (September 7, 2015). Available at https://www.gpo. gov/fdsys/pkg/FR-2015-09-08/pdf/2015-21756.pdf (accessed November 2016)

10. Federal Office of Information and Regulatory Affairs, Unified Agenda, Human Subjects Research Protections released May 18, 2016. Available at http://reginfo.gov/public/do/eAgendaMain (reviewed October 24, 2016) (accessed November 2016)

11. National Academies of Sciences, Engineering, and Medicine. 2015. Optimizing the Nation's Investment in Academic Research: A New Regulatory Framework for the $21^{\text {st }}$ Century: Part 1. Washington, DC: The National Academies Press. Available at http://www.nap.edu/ 21803 (accessed November 2016)

12. National Academies of Sciences, Engineering, and Medicine. 2016. Optimizing the Nation's Investment in Academic Research: A New Regulatory Framework for the $21^{\text {st }}$ Century. Washington, DC: The National Academies Press. Available at http://www.nap.edu/21824 (accessed November 2016)

13. Recommendations on the Notice of Proposed Rulemaking entitled "Federal Policy for the Protection of Human Subjects". Posted 2015 December 28. Available at https://www.regulations.gov/document? $\mathrm{D}=$ HHS-OPHS-2015-0008-0570 (accessed November 2016)

14. Secretary's Advisory Committee on Human Research Protections. Letter to Secretary of Health and Human Services, The Honorable Sylvia Burwell. 2016 July 21, Attachment B. Available at http://www.hhs.gov/ohrp/ sachrp-committee/recommendations/attachment-b-return-individualresearch-results/index.html (accessed November 2016)

15. Secretary's Advisory Committee on Human Research Protections. Letter to Secretary of Health and Human Services, The Honorable Sylvia Burwell. 2015 September 28, Attachment C, p. 2. Available at http://www.hhs.gov/ohrp/sachrp-committee/recommendations/2015september-28-attachment-c/index.html (accessed November 2016)

16. Weil CJ, Mechanic LE, Green T, Kinsinger C, Lockhart NC, Nelson SA, Rodriguez LL, Buccini LD: NCI think tank concerning the identifiability of biospecimens and "omic" data. Genet Med 2013, 15: 997-1003

17. Bevilacqua G, Bosman F, Dassesse T, Höfler H, Janin A, Langer R, Larsimont D, Morente MM, Riegman P, Schirmacher P, Stanta G, Zatloukal K, Caboux E, Hainaut P: The role of the pathologist in tissue banking: European consensus expert group report. Virchows Arch 2010, 456:449-454 